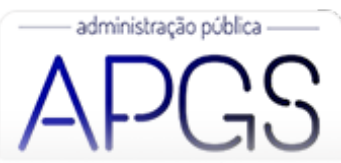

Administração Pública e Gestão Social ISSN: 2175-5787

apgs@ufv.br

Universidade Federal de Viçosa

Brasil

\title{
Investimento em educação diminui a criminalidade: análise dos municípios do Rio Grande do Sul usando econometria espacial
}

Fochezatto, Adelar; Petry, Guilherme; Braatz, Jacó; Romão Marcondes, Henrique

Investimento em educação diminui a criminalidade: análise dos municípios do Rio Grande do Sul usando econometria espacial

Administração Pública e Gestão Social, vol. 13, núm. 4, 2021

Universidade Federal de Viçosa, Brasil

Disponible en: https://www.redalyc.org/articulo.oa?id=351568433014

\section{(c) (1) $\Theta(9)$}

Esta obra está bajo una Licencia Creative Commons Atribución-NoComercial-SinDerivar 3.0 Internacional. 


\title{
Investimento em educação diminui a criminalidade: análise dos municípios do Rio Grande do Sul usando econometria espacial
}

\author{
Investment in education decreases crime: an analysis of Rio Grande do Sul municipalities using spatial econometrics \\ La inversión en educación disminuye la delincuencia: análisis de los municipios de Rio Grande do Sul mediante \\ econometría espacial
}

Adelar Fochezatto

Pontifícia Universidade Católica do Rio Grande do SulRedalyc: https://www.redalyc.org/articulo.oa? PUCRS, Brasil

adelar@pucrs.br

Guilherme Petry

Auditor-Fiscal da Receita Estadual, Brasil

guilhermecp@sefaz.rs.gov.br

Jacó Braatz

Auditor-Fiscal da Receita Estadual, Brasil

jacob@sefaz.rs.gov.br

Henrique Romão Marcondes

PUCRS, Brasil

henrique.marcondes@hotmail.com.br

Recepción: 08 Octubre 2020

Aprobación: 30 Marzo 2021

Publicación: 01 Octubre 2021

\section{Resumo:}

Objetivo da pesquisa: verificar se aumentos de gastos em educação, via vencimentos dos professores, reduz as taxas de criminalidade nos municípios do Rio Grande do Sul.

Enquadramento teórico: teoria econômica do crime (Becker, 1968), que assume a atividade criminal como sendo uma decisão racional, resultado de escolhas entre possíveis benefícios e custos que a mesma pode trazer ao infrator.

Metodologia: análise exploratória de dados espaciais para verificar os padróes geográficos da atividade criminal e modelos de econometria espacial para verificar efeitos de transbordamentos entre os municípios.

Resultados: os resultados obtidos permitem afirmar que um aumento dos gastos públicos em educação nos municípios diminui as taxas de criminalidade dos próprios municípios e dos municípios vizinhos no ano seguinte.

Originalidade: diferentemente de outros trabalhos sobre o tema, que usam gastos agregados, este estudo utiliza apenas os vencimentos dos professores. Além disso, foi usado o gasto público com policiamento em nível de municípios como controle, variável não encontrada em outros trabalhos pela dificuldade de sua obtenção.

Contribuições teóricas e práticas: os resultados indicam que destinar mais recursos aos vencimentos dos professores é um caminho promissor para diminuir a criminalidade nos municípios do Rio Grande do Sul. Outro caminho é adotar políticas que levem ao aumento da renda per capita, ampliando as oportunidades e as chances de inserção no mercado de trabalho.

Palavras-Chave: Educação, Crime, Econometria Espacial.

\section{Abstract:}

Research objective: to verify whether increases in education spending, via teachers' salaries, reduce crime rates in the municipalities of Rio Grande do Sul, in Brazil.

Theoretical framework: economic theory of crime (Becker, 1968), which assumes criminal activity as a rational decision, the result of choices between possible benefits and costs that it can bring to the offender. 
Methodology: exploratory analysis of spatial data to verify the geographical patterns of criminal activity and models of spatial econometrics to verify the effects of overflows between municipalities.

Results: the results obtained allow us to affirm that an increase in public spending on education in the municipalities decreases the crime rates of the municipalities and of the neighboring in the following year.

Originality: unlike other works on the topic, which use aggregate expenses, this study uses only the salaries of teachers. In addition, public spending on policing at the municipal level was used as a control, a variable not found in other studies due to the difficulty of obtaining it.

Theoretical and practical contributions: the results indicate that allocating more resources to teachers' salaries is a promising way to reduce crime in the municipalities of Rio Grande do Sul. Another way is to adopt policies that lead to an increase in per capita income, expanding opportunities and the chances of insertion in the job market.

KEYWORDS: Education, Crime, Spatial Econometrics.

\section{RESUMEN:}

Objetivo de la investigación: verificar si los aumentos del gasto en educación, a través de los salarios de los maestros, reducen las tasas de criminalidad en los municipios de Rio Grande do Sul, en Brasil.

Marco teórico: teoría económica del delito (Becker, 1968), que asume la actividad delictiva como una decisión racional, resultado de elecciones entre posibles beneficios y costos que puede traer al delincuente.

Metodología: análisis exploratorio de datos espaciales para verificar los patrones geográficos de actividad delictiva y modelos econométricos espaciales para verificar efectos de derrame entre municipios.

Resultados: los resultados obtenidos permiten afirmar que un aumento del gasto público en educación en los municipios disminuye las tasas de criminalidad de los propios municipios y de los vecinos en el año siguiente.

Originalidad: a diferencia de otros trabajos sobre el tema, que utilizan gastos agregados, este estudio utiliza únicamente los sueldos de los docentes. Además, se utilizó como control el gasto público en vigilancia a nivel municipal, variable no encontrada en otros estudios por la dificultad de su obtención.

Aportes teóricos y prácticos: los resultados indican que destinar más recursos al salario de los maestros es una forma prometedora de reducir la delincuencia en los municipios de Rio Grande do Sul. Otra forma es adoptar políticas que conduzcan a un aumento del ingreso per cápita, ampliando las oportunidades y las posibilidades de inserción en el mercado laboral.

Palabras Clave: Educación, Crimen, Economometría espacial.

\section{INTRODUÇÃO}

A criminalidade vem sendo uma das principais admoestações das sociedades modernas. Segundo Cerqueira et al. (2017), em 2015, houve, no Brasil, uma taxa de 28,9 homicídios por 100 mil habitantes. Este número representa um aumento de $4 \%$ em relação a 2010, que foi de 27,8 homicídios por 100 mil habitantes. No mesmo período, no Rio Grande do Sul (RS), a taxa aumentou 34,2\%, passando de 19,5 para 26,2 homicídios por 100 mil habitantes.

De acordo com o Relatório Mundial sobre Prevenção da Violência, da Organização Mundial da Saúde (OMS) (2014), uma taxa de dez mortes para cada cem mil habitantes é aceitável, acima disso é tido como uma epidemia. Pode-se afirmar, portanto, que, no cenário brasileiro e do Rio Grande do Sul para o ano de 2015, vivenciava-se uma epidemia. De acordo com o Relatório Mundial sobre a Prevenção da Violência 2014 (OMS, 2014), os homicídios respondem por $2,5 \%$ das mortes ocorridas no mundo, sendo a quarta principal causa de morte.

No entanto, o aumento da criminalidade não ocorreu apenas nos homicídios, mas também em outros tipos de crime. No RS, considerando as estatísticas da Secretaria de Segurança Pública (SSP), o número total de crimes aumentou $7 \%$ entre 2010 e 2015 , passando de 2.677 para 2.865 ocorrências de crimes para cada 100 mil habitantes. Entre os tipos de crimes, os mais frequentes na sociedade gaúcha no período são roubos e furtos, que aumentaram $7,5 \%$ no período, passando de 2.280 para 2.452 ocorrências para cada 100 mil habitantes.

A criminalidade exerce um impacto negativo na qualidade de vida das pessoas, podendo ocasionar perdas patrimoniais, danos emocionais e psíquicos, perda de vida humana, assim como outros custos 
diretos e indiretos com saúde pública, segurança privada, sistema jurídico criminal, redução no turismo e, por conseguinte, uma diminuição da produtividade do país, Cerqueira et al. (2017) apontam também implicações na dinâmica demográfica e no desenvolvimento econômico e social. Ainda em Cerqueira $e t$ al. (2017), alguns dados impressionantes que ilustram esta questão são expostos: se for considerada a faixa etária entre 15 e 29 anos, os jovens do sexo masculino corresponderam a 47,8\% do total de óbitos em 2015 , sendo que na faixa entre 15 e 19 anos a participação masculina é ainda maior, chegando a 53,8\% do total. Em vista disso, o combate à criminalidade tem sido um dos principais fitos no âmbito das políticas públicas.

A concentração de crimes nessa faixa etária naturalmente liga o tema da criminalidade ao da educação. Cunha, Heckman, Lochner e Masterov (2005) evidenciaram que há períodos críticos no ciclo de vida das pessoas, que são cruciais para o desenvolvimento de suas habilidades. Os autores defendem que investimentos na primeira infância para promover a aprendizagem são fundamentais, pois é neste período da vida que a pessoa desenvolve suas primeiras habilidades e as noções de moralidade e civilidade. Essas habilidades potencializam a acumulação de capital humano ao longo do ciclo de vida e promovem melhores oportunidades para o indivíduo, tornando-o menos propenso a cometer crimes (Becker, 2012).

Vários estudos aplicados ao caso brasileiro apontam para a existência de uma relação inversa entre níveis de escolaridade da população e níveis de criminalidade. Fajnzlber e Araújo (2001) encontraram uma relação positiva entre analfabetismo e taxas de homicídios. Kume (2004) e Santos (2009) indicaram uma relação negativa entre escolaridade média (anos de estudo) e criminalidade. Resende (2007) apontou uma relação negativa entre o número de adolescentes entre 15 e 17 anos na escola e homicídios. Scorzafave e Soares (2009) observaram uma relação negativa entre a taxa de matrículas e crimes não pecuniários. Teixeira (2011) assinalou uma relação positiva entre o abandono escolar e homicídios.

O acesso à escola, a retenção do aluno na escola e o seu rendimento escolar dependem de investimentos em recursos humanos e em infraestrutura na área da educação. Por isso, uma via alternativa para estudar a relação entre educação e criminalidade é através dos investimentos públicos na área da educação. Neste sentido, Becker (2012), utilizando dados socioeconômicos dos estados brasileiros, encontrou uma elasticidade negativa entre gastos em educação (defasados em um período) e criminalidade.

Além do enfoque nos investimentos públicos em educação, que este trabalho visa enfatizar, outros indicadores socioeconômicos municipais que podem estar associados com a criminalidade também são utilizados, sendo: i) o percentual de pessoas empregadas formalmente; ii) gastos públicos em segurança; iii) número total de veículos registrados, como proxy de renda per capita; iv) densidade demográfica; e v) como supramencionado, os investimentos públicos em educação.

Este estudo, portanto, busca identificar relaçóes entre o crime e as características socioeconômicas no Rio Grande do Sul em 2015, tratando-se, portanto, de uma análise em cross-section para os 497 municípios do estado. O presente ensaio é embasado mormente na tese de Becker (2012), objetivando verificar especialmente se os investimentos públicos estaduais em educação nos municípios têm influência sobre as taxas locais de criminalidade. Dado que, em geral, os crimes não acontecem de forma aleatória no espaço geográfico, é empregue a metodologia de análise exploratória de dados espaciais e de econometria espacial. O conhecimento de onde ocorrem as concentrações criminais em cada momento do tempo e as relações com as características socioeconômicas dos lugares pode ajudar nas decisões sobre a alocação de recursos escassos na área da segurança.

O trabalho está estruturado como segue: na seção dois é feita uma revisão de trabalhos que se dedicaram a descobrir os possíveis determinantes da criminalidade. Na seção três são apresentados os procedimentos metodológicos. Na seção quatro é feita a análise dos resultados. Por fim, aparecem os comentários conclusivos do trabalho. 


\section{FUNDAMENTOS TEÓRICOS}

Analisando a bibliografia teórica sobre o tema da criminalidade, é possível destacar três dimensões estratégicas como determinantes diretos ou indiretos dos níveis de criminalidade na sociedade: a família, a escola e a cidade. Muitos dos fatores que levam as pessoas a se envolverem com a criminalidade decorrem de situações que aconteceram durante a sua infância. Esses fatores incluem laços fracos com a família e laços fortes com amigos ou colegas antissociais e delinquentes. Farrington (1987) afirma que aumentam as chances de se tornarem infratores jovens que vêm de famílias nas quais a disciplina é inconstante e errática, os pais pouco se preocupam com os filhos e os próprios pais têm problemas com drogas e crimes.

De acordo com Waiselfisz (1998), pelo fato de intervir diretamente na formação das pessoas desde os primeiros anos de vida, a escola é outra dimensão relevante, principalmente pela forte ligação entre a juventude e a violência. Cerqueira (2016) expõe que orientação, supervisão e educação desde a tenra idade são elementos que corroboram com a prevenção ao crime. Soares (2007) diz que a educação é um escudo contra a criminalidade, especialmente no Brasil. Tauchen, Witte e Griesinger (1994) afirmam que, indo à escola ou ao trabalho, reduz-se a probabilidade de os jovens se envolverem em atividades criminosas.

Soares (2004, pp. 160-161) apresenta um resumo sintético dos resultados obtidos em pelo menos 21 trabalhos aplicados, desde efeito positivo, negativo e até não significativo, dependendo da metodologia (dados em painel ou dados de corte transversal) e o tipo de crime (assalto, roubo, homicídio etc.). Vale mencionar que algumas vezes a variável utilizada naqueles foi o índice de pobreza ao invés de uma medida de riqueza.

A urbanização, o tamanho da cidade e a densidade demográfica são variáveis determinantes da criminalidade. Becker (2017) identificou uma relação positiva e significativa entre urbanização e homicídios analisando os dados dos estados brasileiros. De acordo com Beato (1998), a localização dos crimes pode ser explicada pelas oportunidades e pela chance de identificação e captura. Em locais mais densamente povoados há uma diminuição na probabilidade de captura do agente infrator, favorecendo o comportamento delinquente. Grandes cidades oferecem, portanto, mais incentivos para o indivíduo cometer o delito, dado o possível anonimato. Case e Katz (1991) afirmam também que o comportamento de bairros vizinhos afeta substancialmente o comportamento juvenil. Portanto, residir em bairro ou próximo de bairro onde uma grande proporção de jovens está envolvida em crimes aumenta a probabilidade de a pessoa se envolver com a criminalidade.

A taxa de desemprego é outra variável comumente utilizada em trabalhos empíricos como fator associado à criminalidade, especialmente quando se estuda crimes contra o patrimônio. Cerqueira e Lobão (2004), em uma revisão de literatura, expuseram diversos autores que chegaram a resultados com uma relação positiva entre desemprego e criminalidade. De acordo com os mesmos autores, a falta de aptidão para outras atividades também contribui na opção pelo crime. Em Neumayer (2004), a taxa de desemprego mostrou ser uma variável significativa e com sinal positivo para explicar o aumento nas taxas de crime contra a propriedade. Chiricos (1987) utilizou 288 estimações com dados agregados de 63 trabalhos empíricos sobre determinantes do crime e encontrou que o desemprego apresenta sinal positivo e coeficientes estatisticamente significantes em $31 \%$ dos casos e apenas em $2 \%$ este sinal era negativo e o coeficiente estatisticamente significante. Resultados semelhantes são encontrados em Freeman (1995) e Levitt (1997).

Neste trabalho utilizou-se o número total de veículos registrados em cada município como proxy para renda per capita. A renda per capita costuma aparecer em outros trabalhos que estudam o tema, tanto teóricos quanto empíricos. Soares (2004) apresenta um resumo sintético dos resultados obtidos em pelo menos 21 trabalhos aplicados, desde efeito positivo, negativo e até não significativo; dependendo da metodologia (dados em painel ou dados de corte transversal) e o tipo de crime (assalto, roubo, homicídio etc.). Em Tadjoeddin e Murshed (2007), o logaritmo natural da renda per capita aparece com sinal positivo nos modelos de crescimento e de educação propostos pelos autores para explicar o número de atos violentos ou crimes violentos. 
Os gastos estaduais em segurança pública também aparecem neste trabalho visando corroborar com a análise realizada, visando identificar quais fatores estão associados com a criminalidade no RS. Em Loureiro e Carvalho (2007), os gastos em segurança pública apresentaram um sinal positivo na repreensão ao crime. Duenhas, Oliveira e Gelinski (2014), através de uma análise em dados em painel para os municípios brasileiros, chegaram à conclusão que os municípios que mais investem em segurança foram os que mais registraram homicídios no período de 2000 a 2005.

Araujo e Fajnzylber (2000) analisaram as tendências longitudinais e espaciais das taxas de crimes em Minas Gerais e estimaram os determinantes das taxas de criminalidade nas microrregióes do estado. Para tanto, utilizaram variáveis representativas dos níveis educacionais médios, da desigualdade na distribuição de renda, do grau de urbanização, do grau de desorganização social e da participação de jovens na população. Os principais resultados foram: maiores níveis educacionais implicam menores taxas de crime contra a pessoa e maiores taxas de crimes contra a propriedade, a desigualdade de renda encontra-se associada a maiores taxas de homicídios e homicídios tentados e a menores taxas de roubos de veículos, microrregiões mais urbanizadas tendem a ter maiores taxas de estupro e a desorganização social, medida pela taxa de separações, está associada a maiores taxas para todos os crimes. Além disso, uma maior proporção de jovens na população implica maiores taxas de crimes, particularmente aqueles contra a pessoa.

Oliveira (2008) fez uma análise espacial da criminalidade no estado do RS. Para este fim, foi apresentado um modelo econométrico espacial para os determinantes da criminalidade. O modelo seguiu as contribuições da economia do crime, porém, acrescentou as contribuições da abordagem ecológica e as teorias do aprendizado social. No modelo, a criminalidade em cidades pode ser explicada por características locais em que o ambiente, a vizinhança e o histórico do indivíduo afetam a criminalidade. Foram utilizados dados municipais agregados para homicídios, roubos e furtos no ano de 2000. Os testes realizados mostraram a existência de dependência espacial em roubos e furtos e independência espacial em homicídios. No artigo, ficaram destacados os papéis da desigualdade de renda e das aglomerações urbanas como fatores que potencializaram a criminalidade em cidades. Os resultados obtidos mostraram que problemas na estrutura familiar e a ineficiência do ensino afetaram positivamente a criminalidade. Neste artigo, os benefícios do crime e os custos de oportunidade foram divididos, o que permitiu concluir que o crescimento econômico não implicou diretamente no aumento da criminalidade. Isto porque, se houvesse um aumento da renda dos mais pobres, a criminalidade diminuiria.

Kleinschmitt, Wadi e Staduto (2012) analisaram a distribuição espacial dos homicídios no estado do Paraná e a sua relação com as variáveis socioeconômicas, demográficas e de infraestrutura urbana. O método de Análise Exploratória de Dados Espaciais (AEDE) foi utilizado para medir o grau de autocorrelação espacial entre as variáveis estudadas, a partir das características dos municípios do estado. Os resultados demonstraram a existência da autocorrelação espacial das taxas de homicídio entre os municípios paranaenses. Ao considerar os mapas de clusters, verificou-se a presença de quatro grandes clusters do tipo Alto-Alto (AA) e quatro grandes clusters do tipo Baixo-Baixo (BB).

Farias, Figueiredo e Lima (2008) verificaram a existência de clusters entre os municípios mineiros e determinaram um índice de criminalidade municipal, usando variáveis representativas de crimes violentos. Consideraram as similaridades apresentadas entre as taxas de crimes e a existência de possíveis padrões espaciais. Os resultados permitiram inferir que existem sete grupos de municípios distintos em Minas Gerais, conforme nível de criminalidade. Identificaram também que há dependência espacial nas taxas de criminalidade no estado e que existem clusters distintos para crimes violentos contra o patrimônio e contra pessoas.

Enfim, há uma extensa literatura sobre criminalidade e suas possíveis causas. Grande parte dessa literatura baseia-se na economia do crime, proposta por Becker (1968), que aborda a questão como sendo uma atividade racional que apresenta custos e benefícios. Os benefícios seriam os retornos econômicos da atividade criminal e os custos seriam as penalidades impostas caso o infrator seja identificado e capturado. Isto, por 
sua vez, depende da eficiência dos órgãos de segurança e da severidade do sistema penal. Nesta teoria, portanto, desemprego, baixo salário, ineficiência na segurança e sistema penal pouco severo seriam fatores que estimulariam a atividade criminal.

Considerando estudos mais específicos interessados nas relações entre educação e criminalidade, Ahmed, Abdullah e Akhtar (2019) sugerem que através do aumento do status educacional do indivíduo pode-se diminuir a taxa de criminalidade, a pobreza e o desemprego. A educação teria impactos diretos sobre o crime e também impactos indiretos via redução da pobreza e do desemprego.

Em uma perspectiva de longo prazo, Brutti e Montolio (2019) estimam o impacto de uma expansão da pré-escola pública na Espanha na década de 1990 sobre o comportamento criminoso no futuro. Para isso os autores usaram a variação nas taxas de matrícula nas regiões e coortes de nascimento do país e vincularam os dados educacionais a um conjunto de dados de crimes administrativos cometidos na região da Catalunha durante o período de 2009 a 2014. Eles concluem que para a coorte média de nascimentos por município catalão e ano, um aumento de 1 ponto percentual na exposição pré-escolar aos 3 anos resulta em 1,6\% menos ações de crime durante a juventude e a idade adulta jovem.

As relações entre estrutura etária, educação e desemprego com o crime violento são analisadas por Juárez, Urdal e Vadlamannati (2020) para o contexto mexicano. Eles partem da hipótese de que a existência de muitos jovens "ociosos", ou seja, uma oferta abundante de jovens com baixo custo de oportunidade, pode ser um fator que reduz os custos de recrutamento no mundo do crime. Considerando o crime organizado em torno do tráfico de drogas como uma das principais causas do crime e da violência no México, os autores buscam responder se a disponibilidade de grandes grupos de jovens masculinos, baixa escolaridade e alto desemprego juvenil são fatores que contribuem para explicar a variação nas taxas de crimes violentos nos estados mexicanos ao longo do tempo. Usando dados de painel para 32 estados mexicanos no período de 1997 a 2010, eles encontram uma relação positiva e significativa entre desemprego juvenil em estratos de baixa escolaridade e crime violento. A relação entre quantidade de pessoas jovens (grupos de jovens) e crimes violentos não foi significativa.

Butt, Munir, Arif e Zafar (2017) analisaram os efeitos da educação sobre a taxa de criminalidade em países do sul da Ásia, particularmente no Paquistão. O estudo encontrou uma relação positiva entre educação e crime. Os autores justificaram este resultado afirmando que no Paquistão não há oportunidades suficientes para acomodar as pessoas instruídas. As evidências empíricas indicam que as atividades criminosas aumentam os benefícios financeiros mais do que as oportunidades oferecidas às pessoas instruídas. Em vista disso, para diminuir o crime, os autores propõem que haja um esforço para ampliar as oportunidades.

Para o Brasil, Becker (2012) utilizou dados socioeconômicos dos estados brasileiros para estimar a relação entre os investimentos públicos com educação e a criminalidade. Usou um painel de dados dos 26 estados brasileiros e do Distrito Federal, observados entre os anos de 2001 a 2009, compondo uma amostra de 243 observações. A variável "investimento em educação" entrou no modelo com dados defasados em um período. $\mathrm{O}$ estudo encontrou uma elasticidade negativa entre as duas variáveis, indicando que a atuação pública na área de educação pode contribuir para reduzir o crime.

Neste estudo, utiliza-se como referência básica os modelos da economia do crime, inspirados em Becker (1968), juntamente com modelos que exploram mais especificamente os efeitos da educação sobre a criminalidade (Ahmed, Abdullah, \& Akhtar, , 2019; Brutti \& Montolio, 2019; Juárez, Urdal, \& Vadlamannati, 2020; Butt, Munir, Arif, \& Zafar, 2017; Becker, 2012). Na perspectiva da economia do crime, foram revisados vários estudos, que ajudaram a compreender os possíveis fatores que estimulam ou inibem a atividade criminal. Os estudos específicos sobre os efeitos da educação possibilitaram um entendimento mais detalhado de como a educação pode afetar de forma direta e indireta a atividade criminal. Estes modelos serviram de referência para a escolha das variáveis do modelo e para justificar os resultados encontrados. A Figura 1 mostra o esquema analítico e o Quadro 1 as variáveis usadas no modelo deste estudo. 


\section{PROCEDIMENTOS METODOLÓGICOS}

Os crimes não acontecem de forma aleatória no espaço geográfico. O mesmo pode ser dito em relação aos criminosos, pois, em geral, eles vêm de lugares diferentes de onde cometerem o crime. O espaço geográfico, portanto, desempenha um papel vital na compreensão da dinâmica espacial da criminalidade e na definição de estratégias para combatê-la.

Para analisar as relações entre a criminalidade e as variáveis que potencialmente podem influenciá-la, são utilizadas técnicas de autocorrelação e de econometria espacial. A análise de autocorrelação espacial serve para mostrar se o valor de um determinado indicador depende de sua localização nas diferentes unidades espaciais. Em outras palavras, ela mostra se o valor de um determinado indicador segue ou não um padrão espacial aleatório. Quando uma determinada unidade espacial e suas unidades espaciais vizinhas têm comportamentos semelhantes, significa que há autocorrelação espacial positiva e quando elas têm comportamentos diferentes, ela é negativa. Se não há um padrão definido, significa que não há autocorrelação e que, portanto, a distribuição espacial da variável de interesse é aleatória. O principal índice usado para fazer essa mensuração é o I de Moran, que é definido por:

$$
I=\frac{n \sum_{i=1}^{n} \sum_{j=1}^{n} w_{i j}\left(x_{i}-\bar{x}\right)\left(x_{j}-\bar{x}\right)}{\left(\sum_{i=1}^{n} \sum_{j=1}^{n} w_{i j}\right) \sum_{i=1}^{n}\left(x_{i}-\bar{x}\right)^{2}}
$$

em que: . é o índice de correlação espacial global (I de Moran); . é o número de unidades espaciais; $x$. é o valor da variável de interesse na unidade espacial .; $x$. é o valor da variável na unidade espacial .;

é a média da variável .; e $w_{i j}$ é uma matriz de pesos espaciais que indica a relação de contiguidade entre as unidades espaciais . e.. Se . e . compartilham fronteira, então $w_{i j}=1$, senão, $w_{i j}=0$.

O I de Moran é um índice de autocorrelação espacial global, ou seja, considerando todo o conjunto das unidades espaciais. No entanto, é possível que haja autocorrelação espacial entre algumas unidades espaciais e não entre outras, configurando a formação de clusters espaciais. Para verificar a existência de clusters espaciais utiliza-se o indicador Local Indicators of Spatial Association (LISA), o qual é definido por:

$$
I_{i}=\left(x_{i}-\bar{x}\right) \sum_{j=1}^{n} w_{i j}\left(x_{j}-\bar{x}\right)
$$

Para a modelagem econométrica visando estimar o efeito do investimento estadual em educação sobre a criminalidade nos municípios do RS, adota-se a seguinte estratégia: primeiro estima-se um modelo padrão MQO (Mínimos Quadrados Ordinários) sem dependência espacial, ativando os testes para verificar se a especificação poderia ser melhorada incorporando defasagens espaciais. Esses testes indicam também onde incorporar a defasagem espacial, se na variável endógena (spatial lag model - modelo SAR), nos resíduos (spatial error model - modelo SEM) ou em ambas (modelo SAC). Para essas três situações, os modelos apresentam, respectivamente, as seguintes especificações: 


$$
\begin{gathered}
y=\rho W y+X \beta+\varepsilon \\
y=X \beta+\mu \\
\mu=\lambda W \mu+\varepsilon \\
y=X \beta+(I-\lambda W)^{-1} \varepsilon \\
y=\rho W y+X \beta+(I-\lambda W)^{-1} \varepsilon
\end{gathered}
$$

onde: .é um vetor de valores da variável dependente; .é uma matriz de valores das variáveis independentes; . é um vetor de erros da regressão; . é um vetor de parâmetros associados às variáveis independentes; $\rho$ é um coeficiente auto-regressivo que mede a proporção da variação total da variável dependente que é explicada pela autocorrelação espacial dessa variável (pela média dos seus valores nas unidades espaciais vizinhas); . é uma matriz de pesos espaciais (matriz de vizinhança); . é um coeficiente auto-regressivo para os erros da regressão que mede a influência dos resíduos das unidades espaciais vizinhas; . é um vetor de erros espacialmente autocorrelacionados; . é uma matriz identidade.

A criminalidade possui um padrão de concentração espacial e pode influenciar os municípios que estão na vizinhança. Por isso justifica-se o uso da econometria espacial, que tem por característica levar em consideração os efeitos de unidades geográficas vizinhas. $\mathrm{O}$ indicador de criminalidade utilizado neste trabalho apresentou uma alta autocorrelação espacial positiva, justificando o uso da econometria espacial.

Além dos investimentos públicos em educação (variável de interesse), outros possíveis fatores associados à criminalidade apontados pela literatura pertinente serão utilizados como controles no modelo econométrico. Essas outras variáveis são comuns em estudos de economia do crime: empp é um indicador do ambiente no mercado de trabalho local, veiregpc é um indicador de renda da população local, dpop é um indicador de densidade populacional e de perfil urbano local e tvspc é um indicador de segurança ou de capacidade de dissuasão ou combate ao crime na localidade. O quadro abaixo apresenta a lista de variáveis usadas com as respectivas fontes das informações e os sinais esperados para os coeficientes das mesmas nas regressões. $\mathrm{O}$ Quadro 1 apresenta uma descrição das variáveis utilizadas neste estudo.

Quadro 1 - Variáveis utilizadas com as respectivas fontes e sinais esperados

\begin{tabular}{|l|l|l|l|l|}
\hline Sigla & Descriçä́ & Fonte & Sinal esperado & Ano \\
\hline totcrpc & Número total de crimes por 1000 habitantes em cada municipio & Secretaria de Segurança Pública do Rio Grande do Sul & Variável endógena & 2015 \\
\hline empp & Porcentagem de pessoas empregadas formalmente (inverso do desemprego) & Ministério do Trabalho e Emprego & Negativo \\
\hline tvepc & $\begin{array}{l}\text { Investimentos públicos em educaçăo (escolas) por 1000 habitantes em cada } \\
\text { municipio }\end{array}$ & Secretaria da Fazenda do Rio Grande do Sul & Negativo \\
\hline tvspc & $\begin{array}{l}\text { Gastos públicos em segurança (policiamento) por 1000 habitantes em cada } \\
\text { municipio }\end{array}$ & Secretaria da Fazenda do Rio Grande do Sul & 2014 \\
\hline veiregpc & $\begin{array}{l}\text { Número total de veículos registrados por 1000 habitantes em cada município } \\
\text { (proxy de renda per capita) }\end{array}$ & Fundaçăo de Economia e Estatística do Rio Grande do Sul & Negativo & Ambíguo \\
\hline dpop & Densidade populacional do município & Fundaçăo de Economia e Estatística do Rio Grande do Sul & Positivo \\
\hline
\end{tabular}

Fonte: Elaborado pelos autores.

A literatura relacionada ao tema deste estudo diz que a educação pode reduzir o índice de criminalidade dos municípios de forma direta e também através do crescimento econômico e redução do desemprego. A partir dessa literatura elaborou-se o esquema teórico-analítico da Figura 1, que foi usado para selecionar as variáveis do modelo e justificar os resultados encontrados neste trabalho. Esse esquema mostra os possíveis mecanismos de causa e efeito presentes no modelo estimado. Ele mostra como um aumento de gastos em educação, neste caso um aumento dos vencimentos dos professores por mil habitantes do município, pode levar a uma redução da criminalidade no município. 
Figura 1 - Esquema teórico-analítico usado neste trabalho



Fonte: Elaborado pelos autores.

O aumento de gastos em educação pode aumentar a motivação e o esforço dos professores no sentido de proporcionar um ensino de melhor qualidade, diminuindo a evasão escolar. Também pode aumentar o interesse dos professores em aumentar a captação e a retenção de alunos nas escolas. Isto pode diminuir o tempo dos alunos fora da escola, reduzindo a sua exposição ao crime. Além disso, aumenta o aprendizado, melhorando as perspectivas de inserção no mercado de trabalho e de aumento do seu rendimento. Isso, por sua vez, pode reduzir a motivação ao crime. Todos esses efeitos combinados acabam gerando um efeito positivo sobre o crescimento das economias locais, aumentando as oportunidades.

A Tabela 1 abaixo apresenta as estatísticas descritivas das variáveis utilizadas no estudo, referentes ao ano de 2015 .

Tabela 1 - Estatísticas descritivas das variáveis usadas no modelo, 2015

\begin{tabular}{lllll}
\hline Variáveis & Média & Mediana & Mínimo & Máximo \\
\hline Totcrpc & 15,49 & 13,65 & 1,29 & 77,53 \\
Dpop & 93,09 & 23,27 & 1,48 & $3.091,92$ \\
veiregpc & 552,79 & 553,20 & 227,96 & 817,41 \\
Empp & 26,79 & 22,51 & 6,63 & 97,29 \\
Tvepc & 323,88 & 309,74 & 67,31 & 927,43 \\
TvSpc & $6.924,31$ & $5.681,62$ & 0,00 & $44.621,33$ \\
\hline
\end{tabular}


Fonte: Elaborado pelos autores.

Para avaliar a presença ou não de dependência espacial é preciso usar uma matriz de pesos espaciais. A matriz usada neste estudo foi a de vizinhança de contiguidade do tipo Rainha de primeira ordem (Queen). Entre as opções testadas, esta matriz foi a que originou o maior valor do I de Moran. Além desta matriz, foram testadas matrizes de vizinhos mais próximos (3, 4 e 5) e de distância $(68 \mathrm{Km}$ e $100 \mathrm{Km})$. Para maiores detalhes sobre os tipos de matrizes de pesos espaciais, ver Almeida (2012).

Para analisar o efeito do gasto público sobre a criminalidade nos municípios do RS, primeiro foi estimado um modelo padrão com a seguinte especificação:

$$
\text { totcrpc }_{i}=\beta_{0}+\beta_{1} * \text { tvepc }_{i}+\beta_{2} * t v s p c_{i}+\beta_{3} * d p o p_{i}+\beta_{4} * \text { empp }_{i}+\beta_{5} * \text { veiregpc } c_{i}+\varepsilon_{i}
$$

Onde: totcrpc. é o indicador de criminalidade do município .; tvepc. é o indicador de gasto público em educação por mil habitantes no município . (referente ao ano anterior); tvspc. é o indicador de gasto público em segurança por mil habitantes no município .(referente ao ano anterior); dpop. é a densidade demográfica do município .; empp. é a porcentagem de pessoas empregadas em emprego formal no município .; veiregpc. é o número de veículos registrados por mil habitantes no município $i ; \beta$., $\beta$., ..., $\beta$. são os coeficientes estimados associados às variáveis independentes; $\varepsilon$. é um termo de erro da regressão para o município ..

Para tvepc. e para tvspc. foram usados os valores defasados em um ano para diminuir o problema de endogeneidade e também porque os efeitos destas variáveis sobre a criminalidade podem não ser imediatos. Foram testadas outras defasagens e diferentes combinações de defasagens para as variáveis tvepc. e tvspc. e os resultados se mantiveram praticamente inalterados, mas com ajuste um pouco melhor com uma e cinco defasagens. Assim, optou-se por considerar uma defasagem por ser a utilizada por Becker (2012), trabalho este que é utilizado como referência neste artigo.

Foi testada também a variável investimentos na função educação por município no lugar da variável tvepc, mas ela se mostrou não significativa e produziu um ajuste pior. Não foi possível usar ambas no mesmo modelo devido ao problema de multicolineridade. A Tabela 2 mostra os resultados dessa estimação, incluindo um diagnóstico de dependência espacial.

Ao fazer um diagnóstico da regressão, percebe-se que o modelo não possui multicolinearidade (condition number $=17,61$ ), mas, por outro lado, apresenta problemas de não normalidade dos resíduos (teste JarqueBera com p-valor $=0,00$ ) e de heterocedasticidade (teste Breusch-Pagan com p-valor $=0,00$ ), resultado comum quando existe dependência espacial nos dados.

No diagnóstico de dependência espacial existem seis testes. No primeiro deles, tem-se um I de Moran de 0,27 e altamente significativo, rejeitando a hipótese nula de aleatoriedade espacial da criminalidade e indicando a existência de autocorrelação espacial nos resíduos. Os demais testes servem para mostrar qual a melhor opção em termos de inclusão de variáveis defasadas espacialmente. Pode-se ver que os dois testes simples "Lagrange Multiplier (lag)" e "Lagrange Multiplier (error)" são significativos, indicando a presença de dependência espacial. Os testes robustos ajudam a identificar que tipo de dependência espacial pode estar ocorrendo. Nestes, o teste "Robust LM (lag)" é significativo e o "Robust LM (error)" não é significativo. Portanto, o mais indicado é utilizar um modelo que incorpore este tipo de dependência espacial. Trata-se, neste caso, de dependência espacial na variável endógena, ou seja, as taxas de criminalidade nos municípios estão associadas às taxas de criminalidade nos municípios vizinhos. $\mathrm{O}$ modelo indicado neste caso é o SAR. Para mais detalhes sobre a interpretação dos testes e procedimentos para a escolha do modelo espacial mais apropriado, ver Almeida (2012).

Ao utilizar o modelo SAR, a qualidade do ajuste do modelo melhorou: o R. aumentou e AIC diminuiu. No entanto, continuou ocorrendo o problema de heteroscedasticidade. Com isso, foi necessário usar uma 
versão que permitisse a correção desse problema, o que foi feito através de uma estimação pelo método de mínimos quadrados ponderados espacialmente em dois estágios (Spatially weighted two stage least squares HET). Neste caso, as defasagens espaciais de todas as variáveis explicativas foram usadas como instrumentos. A Tabela 2 apresenta os resultados dos três tipos de modelos estimados MQO, SAR e HET.

\section{RESULTADOS E DISCUSSÃO}

O primeiro aspecto a ser considerado na análise dos resultados é o padrão de distribuição espacial das variáveis de interesse. Esta visualização permite verificar se há semelhanças ou diferenças na distribuição espacial destas variáveis. Neste caso, apresenta-se na Figura 2 os mapas temáticos de dez intervalos iguais. Na parte superior são mostrados os investimentos com educação por 1000 habitantes nos municípios do RS no ano de 2014 . Na parte inferior é mostrado o total de crimes por 1000 habitantes nos municípios do RS no ano de 2015.

Figura 2 - Mapas temáticos de Investimentos em educação por 1000 habitantes

(2014) e de crimes por 1000 habitantes (2015) nos municípios do RS

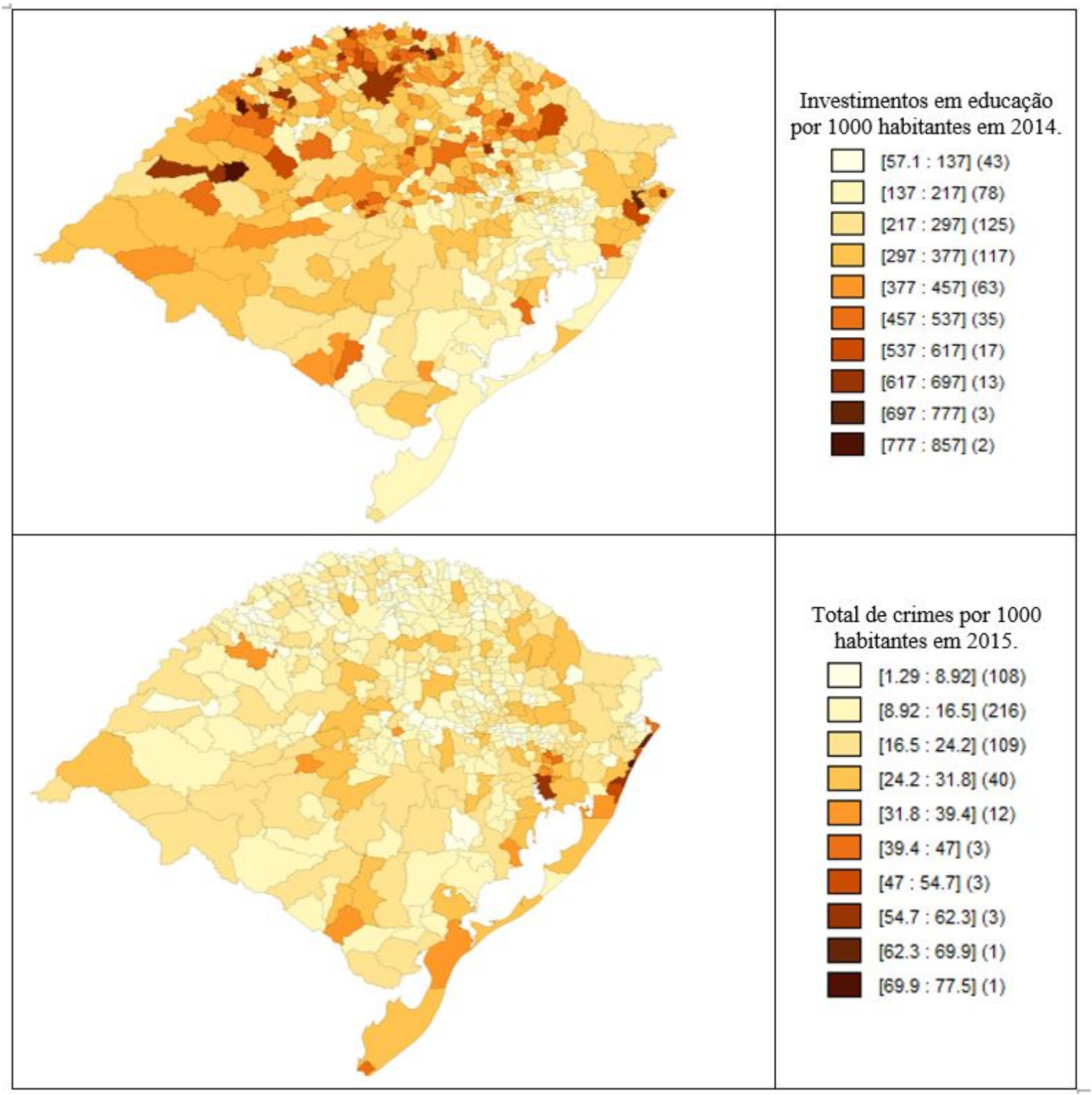


Fonte: Elaborado pelos autores. Nota: mapas de dez intervalos iguais.

Em linhas gerais, pode-se dizer que os padrões espaciais dessas duas variáveis são distintos. Onde há mais gasto com educação há menos crimes e vice-versa. Os mapas apenas mostram os padrões de distribuição, mas não permitem afirmar nada sobre relações de causa e efeito. Para isso seria necessário fazer um teste de causalidade.

O segundo aspecto a ser considerado é o padrão espacial da criminalidade e dos gastos em educação. Na Figura 3 aparecem dois diagramas mostrando, respectivamente, o I de Moran univariado e multivariado. $\mathrm{O}$ primeiro caso (a) mostra a relação entre o indicador de criminalidade nos municípios e o mesmo indicador nos municípios vizinhos (totcrpc e $W_{-}$totcrpc). Pode-se perceber que esse indicador apresenta forte autocorrelação espacial positiva (0,3825). No segundo caso, quando se analisa a autocorrelação espacial do total de crimes com o gasto em educação (tvepc e $W_{-}$totcrpc), percebe-se uma relação negativa $(-0,1466)$.

Figura 3 - Autocorrelação espacial global dos indicadores de criminalidade (totcrpc) e de gasto em educação (tvepc) nos municípios do RS, 2015

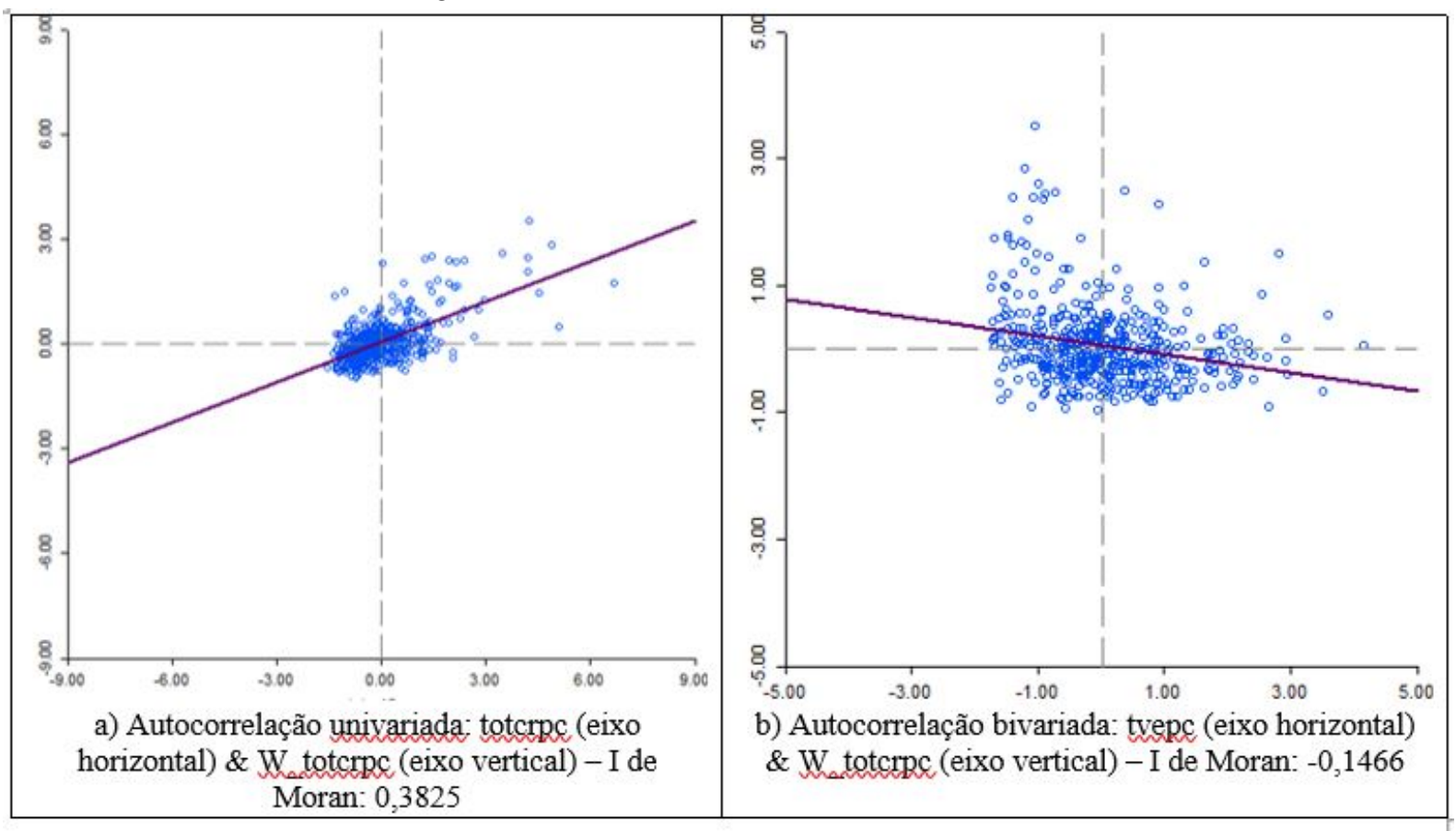

\footnotetext{
Fonte: Elaborado pelo autor. Nota: W_totcrpc corresponde ao comportamento do indicador de criminalidade nos municípios vizinhos.
}

O I de Moran univariado e bivariado da Figura 2 indica que a criminalidade é um fenômeno que apresenta dependência espacial. Este comportamento justifica o uso de econometria espacial, pois ela incorpora a este comportamento através das matrizes de peso espaciais.

O I de Moran é um indicador global de autocorrelação e seus resultados podem variar de local para local. Pode haver autocorrelação positiva alta em alguns locais (combinação high-high) e baixa em outros (combinação low-low), como pode também haver autocorrelação negativa (combinações high-low ou lowhigh). Para verificar a presença de clusters espaciais entre esses indicadores, foi calculado o indicador de autocorrelação espacial local (LISA). A Figura 3 mostra que existem alguns clusters do tipo alto-alto (alta criminalidade associada à alta criminalidade nos municípios vizinhos), principalmente nas regiões Metropolitana de Porto Alegre e Litoral Norte. Na Figura 4, é possível verificar, também, que existem alguns clusters espaciais do tipo baixo-baixo (baixa criminalidade associada à baixa criminalidade nos municípios vizinhos), localizados ao norte do RS. 
O mapa inferior da Figura 4 mostra os clusters bivariados entre criminalidade e gasto em educação. É possível verificar que há uma predominância de clusters do tipo baixo-alto (baixo gasto em educação per capita associado à alta criminalidade nos municípios vizinhos), principalmente nas regióes Metropolitana de Porto Alegre e Litoral Norte. No Norte do RS aparecem alguns clusters espaciais do tipo alto-baixo (alto gasto em educação per capita associado à baixa criminalidade nos municípios vizinhos).

Figura 4 - Autocorrelação espacial local dos indicadores de criminalidade (totcrpc) e de gasto em educação (tvepc) nos municípios do RS, 2015

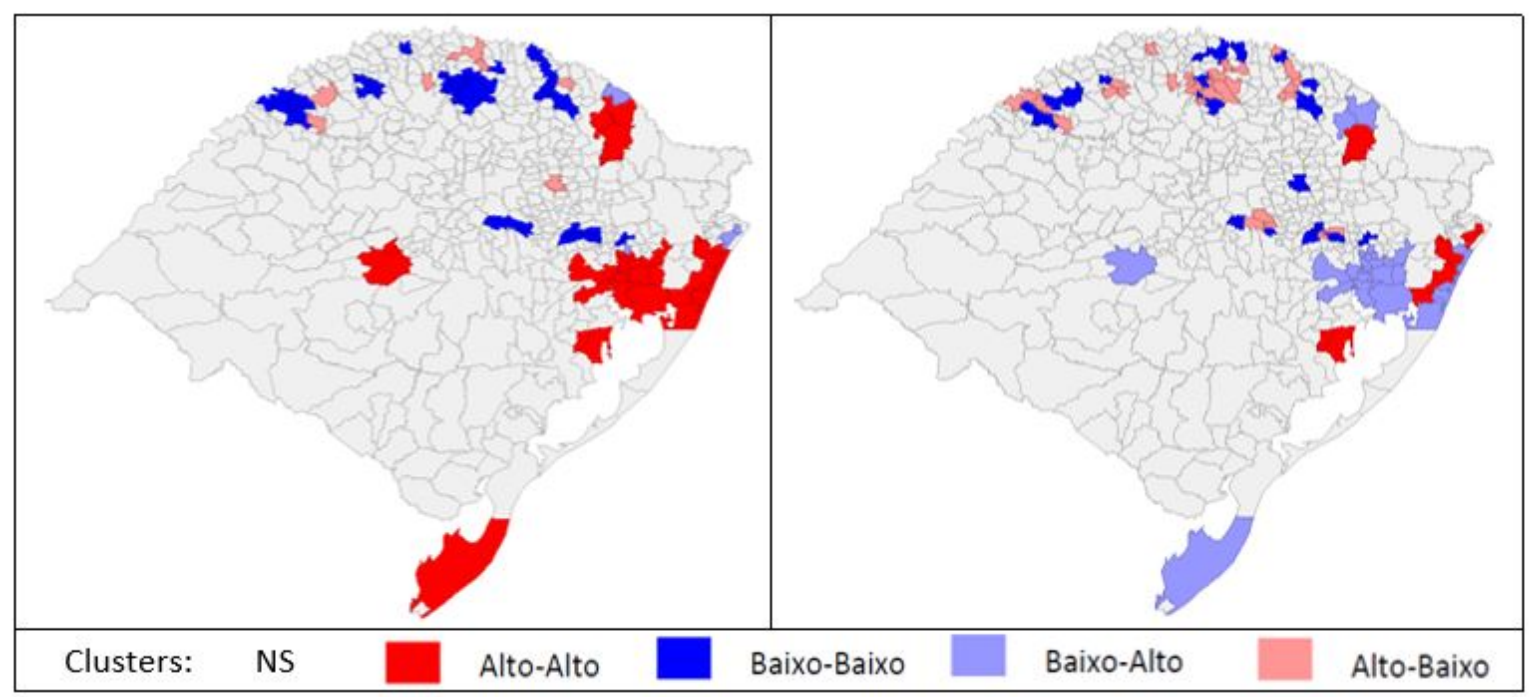

Fonte: Elaborado pelo autor através do software Geoda 1.10.

A Tabela 2 apresenta os resultados dos modelos estimados. A análise é feita apenas para os resultados do modelo (HET). Considerando que se trata de uma análise de dados em cross-section, os resultados mostram que o modelo tem uma boa capacidade explicativa da criminalidade (Pseudo R. de 0,4538). Os coeficientes das variáveis independentes são todos significativos, com exceção do lambda (coeficiente de dependência espacial nos resíduos). Os sinais dos coeficientes, em sua maioria, confirmaram as hipóteses levantadas na Tabela 1. Apresentaram sinais contrários aos esperados a porcentagem de empregados na população (empp) e os gastos com segurança defasados em um período (tvspc). Um valor positivo e significativo do coeficiente de dependência espacial (rho) indica que a atividade criminal não se distribui de forma aleatória no espaço geográfico. Pode-se dizer que municípios com altas taxas de criminalidade são cercados por municípios também com altas taxas de criminalidade e que municípios com baixas taxas de criminalidade são cercados por municípios também com baixas taxas de criminalidade.

O efeito positivo da densidade populacional (dpop) sobre a taxa de criminalidade está de acordo com o modelo da economia do crime (Becker, 1968). Por um lado, um lugar mais denso de pessoas aumenta as oportunidades para a atividade criminal e, por outro lado, diminui as chances do infrator ser identificado e capturado. O efeito negativo do número total de veículos registrados por 1000 habitantes (veicrepc), proxy de renda per capita, pode ser justificado pelo fato que os locais com esta caraterística apresentam mais oportunidades de inserção no mercado de trabalho. Estes resultados estão alinhados com os de Ahmed et al. (2019), Brutti e Montolio (2019), Juárez et al. (2020) e Butt et al. (2017).

A variável de interesse apresentou o sinal esperado, encontrado também por Becker (2012). Com isso, pode-se afirmar que os investimentos em educação nos municípios (despesas em recursos humanos) no período anterior tendem a ocasionar uma queda na criminalidade nos municípios no período corrente. Em termos mais precisos, o aumento de uma unidade nos investimentos em educação por mil habitantes nos 
municípios no período anterior (2014), ocasionaram uma redução de 0,0053 crimes por mil habitantes nos municípios no período corrente (2015).

Conforme modelo teórico usado neste estudo, pode-se dizer que o aumento dos gastos em educação impacta positivamente a qualidade da educação e negativamente a evasão escolar. A melhoria da qualidade da educação pode diminuir o estímulo ao crime, pois tende a melhorar a perspectiva de inserção e de retorno no mercado de trabalho. Além disso, o fato de estar mais tempo na escola, diminui o tempo de exposição ao crime, já que a sala de aula é um ambiente relativamente mais seguro. Esses efeitos combinados acabam impactando positivamente o ambiente social e o crescimento econômico local (Ahmed et al., 2019; Brutti \& Montolio, 2019; Juárez et al., 2020; Butt et al., 2017; Becker, 2012).

Tabela 2 - Resultados da estimação por Mínimos Quadrados Ordinários

\begin{tabular}{|c|c|c|c|c|c|c|}
\hline \multirow{2}{*}{ Variáveis } & \multicolumn{2}{|l|}{ MQO } & \multicolumn{2}{|l|}{ SAR } & \multicolumn{2}{|l|}{ HET } \\
\hline & coeficiente & p valor & coeficiente & p valor & coeficiente & p valor \\
\hline const & $\begin{array}{l}21,7805 \\
(2,2831)\end{array}$ & 0,0000 & $\begin{array}{l}8,8177 \\
(2,1994)\end{array}$ & 0,0000 & $\begin{array}{l}8,1156 \\
(4,4337)\end{array}$ & 0,0672 \\
\hline dpop & $\begin{array}{l}0,0080 \\
(0,0011)\end{array}$ & 0,0000 & $\begin{array}{l}0,0048 \\
(0,0010)\end{array}$ & 0,0000 & $\begin{array}{l}0,0045 \\
(0,0014)\end{array}$ & 0,0013 \\
\hline empp & $\begin{array}{l}0,1430 \\
(0,0274)\end{array}$ & 0,0000 & $\begin{array}{l}0,1342 \\
(0,0244)\end{array}$ & 0,0000 & $\begin{array}{l}0,1302 \\
(0,0257)\end{array}$ & 0,0000 \\
\hline veicrepc & $\begin{array}{l}-0,0197 \\
(0,0037)\end{array}$ & 0,0000 & $\begin{array}{l}-0,0119 \\
(0,0033)\end{array}$ & 0,0000 & $\begin{array}{l}-0,0117 \\
(0,0044)\end{array}$ & 0,0078 \\
\hline tvepc(t-1) & $\begin{array}{l}-0,0102 \\
(0,0028)\end{array}$ & 0,0004 & $\begin{array}{l}-0,0057 \\
(0,0025)\end{array}$ & 0,0247 & $\begin{array}{l}-0,0053 \\
(0,0027)\end{array}$ & 0,0443 \\
\hline tvspc(t-1) & $\begin{array}{l}0,0005 \\
(0,0000)\end{array}$ & 0,0000 & $\begin{array}{l}0,0004 \\
(0,0000)\end{array}$ & 0,0000 & $\begin{array}{l}0,0004 \\
(0,0000)\end{array}$ & 0,0000 \\
\hline rho & - & - & $\begin{array}{l}0,5281 \\
(0,0462)\end{array}$ & 0,0000 & $\begin{array}{l}0,5658 \\
(0,1617)\end{array}$ & 0,0005 \\
\hline lambda & - & - & - & - & $\begin{array}{l}-0,0622 \\
(0,2382)\end{array}$ & 0,7942 \\
\hline R2 ajustado & 0,2850 & & 0,4455 & & 0,4538 & \\
\hline $\mathrm{F}$ & 40,4583 & 0,0000 & & & & \\
\hline $\mathrm{AIC}$ & 3459,67 & & 3370,28 & & & \\
\hline Breusch-Pagan & 115,3167 & 0,0000 & 84,2159 & 0,0000 & & \\
\hline $\mathrm{N}$ & 496 & & 496 & & 496 & \\
\hline
\end{tabular}

Fonte: Elaborado pelo autor. Modelos estimados com os softwares Geoda 1.10 (MQO e SAR) e SpaceGeoda (HET). Instrumentos do modelo HET: W_dpop, W_empp, W_tvspc(t-1), W_tvepc(t-1), W_veicrepc, em que o W indica que a variável está defasada espacialmente.

A partir desses resultados, pode-se calcular o aumento dos investimentos em educação por mil habitantes nos municípios, necessário para reduzir em uma unidade o número de crimes por mil habitantes. Este valor é de $\mathrm{R} \$ 188,68$ a cada mil habitantes por ano $(1 / 0,0053=188,68)$, ou seja, um aumento de $\mathrm{R} \$ 188,68$ na educação por mil habitantes, correspondem à redução de um crime por mil habitantes.

Assim, considerando os valores médios da Tabela 1 , se o gasto médio em educação por mil habitantes nos municípios aumentasse de $\mathrm{R} \$ 323,88$ para $\mathrm{R} \$$ 512,56, o número de crimes por mil habitantes nos municípios no ano seguinte passaria de 15,49 para 14,49. Em termos absolutos, para o estado como um todo, isso significaria um aumento de $\mathrm{R}$ \$2.121.711,00 nos investimentos públicos anuais em educação para uma diminuição anual de 11.245 crimes.

De modo análogo, seria necessário gastar R \$2.399,81 (1/0,0004167 =2.399,81) em segurança pública por ano a cada mil habitantes para se reduzir em uma unidade o número de crimes no ano seguinte. Ratificando, desse modo, o impacto superior na redução da criminalidade ao se investir em educação em cotejo ao dispendido em segurança pública. 


\section{CONSIDERAÇÕES FINAIS}

O objetivo deste estudo foi verificar se os investimentos públicos em educação nos municípios influenciam as taxas municipais de criminalidade. Dado que, em geral, os crimes não acontecem de forma aleatória no espaço geográfico, foi utilizada a metodologia de análise exploratória de dados espaciais e de econometria espacial.

$\mathrm{Na}$ análise exploratória de dados espaciais foi possível verificar que a criminalidade apresenta autocorrelação espacial positiva. Isso significa dizer que os locais de alta criminalidade são cercados de municípios também com alta criminalidade. Também pode-se afirmar que os locais de baixa criminalidade são rodeados de municípios também com baixa criminalidade. Analisando a relação espacial entre investimentos em educação e criminalidade, verificou-se uma associação negativa, ou seja, os locais de baixo gasto em educação são cercados de municípios com alta criminalidade. Raciocínio inverso pode ser feito para os locais com alto gasto em educação.

Ainda na análise exploratória, foi calculada a correlação espacial local (LISA). Foram encontrados clusters do tipo alto-alto, principalmente nas regióes Metropolitana de Porto Alegre e Litoral Norte. Por outro lado, foram encontrados alguns clusters espaciais do tipo baixo-baixo, localizados ao norte do estado. Foi calculada também a correlação espacial local bivariada entre investimentos em educação e criminalidade. Verificou-se uma predominância de clusters do tipo baixo-alto, principalmente nas regiões Metropolitana de Porto Alegre e Litoral Norte. No Norte do RS aparecem alguns clusters espaciais do tipo alto-baixo.

Por fim, os resultados do modelo econométrico espacial indicaram que aumentos de investimentos em educação nos municípios (despesas com recursos humanos) no período anterior tendem a ocasionar uma queda na criminalidade nos municípios no período corrente. Com os resultados obtidos foi possível calcular que seria necessário aumentar em $\mathrm{R} \$ 188,68$ os investimentos em educação por mil habitantes nos municípios para reduzir um crime por mil habitantes. Considerando o Estado como um todo, foi possível calcular que o valor referido acima significaria um aumento de $\mathrm{R} \$ 2.121 .711,00$ nos investimentos públicos anuais em educação para uma diminuição anual de 11.245 crimes.

Enfim, os resultados deste estudo indicam que destinar mais recursos para a educação, especialmente para aumentar os vencimentos dos professores, é um caminho promissor para diminuir a criminalidade nos municípios do Rio Grande do Sul. Outra medida factível seria adotar políticas que levem a um aumento da renda per capita, ampliando as oportunidades e aumentando as chances de inserção no mercado de trabalho. Para chegar a esses resultados, o estudo contou com dados de gastos com vencimentos de professores por escolas nos municípios e de gastos com vencimentos de policiais nos municípios. Em trabalhos futuros, a análise pode ser melhorada em pelo menos dois aspectos: usando um período de tempo mais longo, possibilitando o uso de modelos de dados em painel espacial, e usar outras variáveis de gastos em educação, como em infraestrutura e acessibilidade escolar.

\section{REFERÊNCIAS}

Ahmed, B., Abdullah, U., Akhtar, S. (2019). The Relationship between Education and Crime Analysis (1991-2016): A Case Study of Pakistan. International Journal of Humanities, Arts and Social Sciences, 5(5), 171-182.

Almeida E. S., Haddad, E. A., Hewings, G. J. D. (2005). The spatial pattern of crime in Minas Gerais: An exploratory analysis. Economia Aplicada, São Paulo, 9(1), 39-55.

Almeida. E. (2012). Econometria Espacial Aplicada. Campinas: Alínea.

Araujo, A. F. Jr., Fajnzylber, P. (2000). Crime e Economia: Um Estudo das Microrregiões Mineiras. Revista Econômica do Nordeste, Fortaleza, 31(especial), 630-659.

Beato, C. C. (1998). Determinantes da criminalidade em Minas Gerais. Revista Brasileira de Ciências Sociais, São Paulo, 13(37), 1-16.

Becker, G. S. (1968). Crime and Punishment: An Economic Approach. Journal of Political Economy, 76(2), 169-217. 
Becker, K. L. (2012). Uma análise econômica da relação entre a educação e a violência. Esalq/USP: $2012.76 \mathrm{f}$. Tese (Doutorado em Ciências) -Escola Superior de Agricultura Luiz de Queiroz, Universidade de São Paulo, Piracicaba.

Becker, K. L., Kassouf, A. L. (2017). Uma análise do efeito dos gastos públicos em educação sobre a criminalidade no Brasil. Economia e Sociedade, 26(1), 215-242.

Brutti, Z., Montolio, D. (2019). Preventing Criminal Minds: Early Education Access and Adult Offending Behavior. The Barcelona Institute of Economics (IEB), IEB Working Paper n. 2019/02.

Butt, M., Munir, S., Arif, S., Zafar, F. (2017). The Influence of Academic Education on Crime Rate within South Asia: A Case Study from Pakistan. Advances in Social Sciences Research Journal, 4(13), 76-85.

Case, A. C., Katz, L. (1991). The Company You Keep: The Effects of Family and Neighborhood on Disadvantaged Youths. NBER Working Papers n. 3705, Massachusetts.

Cerqueira, D., Lobão, W. (2004). Determinantes da criminalidade: arcabouços teóricos e resultados empíricos. Dados, 47(2), 233-269.

Cerqueira, D. (2016). Trajetórias individuais, criminalidade e o papel da educação. Boletim de Análise PolíticoInstitucional/IPEA, 9, 27-35.

Chiricos, T. G. (1987). Rates of crime and unemployment: An analysis of aggregate research evidence. Social problems, $34(2), 187-212$.

Cunha, F., Heckman, J.J., Lochner, L., Masterov, D.V. (2005). Interpreting the Evidence on Life Cycle Skill Formation. NBER Working Paper Series n.11331, Cambridge.

Duenhas, R. A., Oliveira, G. F., Gelinski, E. G. Júnior (2014). Educação, segurança pública e violência nos municípios brasileiros: uma análise de painel dinâmico de dados. Publicatio UEPG: Ciências Sociais Aplicadas, Ponta Grossa, 22(2), 179-191.

Fajnzylber, P., Araújo, A. Jr. (2001). Violência e criminalidade. In: Lisboa, M. B. Menezes Filho, N. A. (ed.) Microeconomia e Sociedade no Brasil. Rio de Janeiro: Contracapa Editora.

Farias, C. A., Figueiredo, A. M., \& Lima, J. E. (2008). Dependência Espacial e Análise de Agrupamento de Municípios para Diferentes Tipos de Crime em Minas Gerais. Reuna, 13(3), 67-83.

Farrington, D. P. (1987). Predicting Individual Crime Rates. Crime and Justice, Chicago, 9, 53-101.

Freitas, T. A., Cadaval, A. F., Gonçalves, G. A. (2015). A estimação de um índice geral de criminalidade para os municípios do Rio Grande do Sul - Igcrime RS. Anais do Encontro Nacional da Associação Brasileira de Estudos Regionais e Urbanos, Curitiba, PR, Brasil, 13.

Juárez, N. C., Urdal, H., Vadlamannati, K. C. (2020). The significance of age structure, education, and youth unemployment for explaining subnational variation in violent youth crime in Mexico. Conflict Management and Peace Science, 00(0), 1-25.

Kleinschmitt, S. C., Wadi, Y. M., Staduto, J. A. (2012). Análise espacial dos homicídios no Estado do Paraná. Revista REDES, 17(3), 257-290.

Kleinschmitt, S. C., Wadi, Y. M., Staduto, J. A. (2010). Evolução espaço-temporal dos homicídios no Estado do Paraná. REBESP, 4(3), 16-27.

Kume, L. (2004). Uma Estimativa dos Determinantes da Taxa de Criminalidade Brasileira: Uma Aplicação em Painel Dinâmico. Anais do Encontro Nacional de Economia, João Pessoa, PB, Brasil, 32.

Levitt, S. D. (1997). Using electoral cycles in police hiring to estimate the effect of policeon crime. The American Economic Review, 87(3), 270-290.

Loureiro, A. O. F., Carvalho, J. R. de A. (2007). O impacto dos gastos públicos sobre a criminalidade no Brasil. In: Hermanns, K., Arraes, R. A. (org.) Desigualdades e políticas regionais. Fortaleza: Fundação Konrad Adenauer, 165-193.

Oliveira, C. A. (2008). Análise espacial da criminalidade no Rio Grande do Sul. Revista de Economia, 34(3), 35-60.

Organização Mundial da Saúde. (2014). Relatório Mundial sobre Prevenção da Violência. Genebra. 




Resende, J. P. (2007). Crime social, castigo social: O efeito da desigualdade de renda sobre as taxas de criminalidade nos grandes municipios brasileiros. 2007. 83 p. Dissertação (Metrado em Economia) - CEDEPLAR, Universidade Federal de Minas Gerais, Belo Horizonte.

Santos, M. J. (2009). Dinâmica temporal da criminalidade: mais evidências sobre o efeito inércia nas taxas de crimes letais nos estados brasileiros. Revista Economia, Brasília/DF, 10(1), 170-194.

Scorzafave, L. G., Soares, M. K. (2009). Income inequality and pecuniary crimes. Economics Letters, 104(1), 40-42.

Soares, R. R. (2004). Development, crime and punishment: accounting for the international differences in crime rates. Journal of Development Economics, 73(1), 155-184.

Soares, S. S. D. (2007). Educaşão, um escudo contra o homicídio? TD 1298, Brasília: IPEA.

Tadjoeddin, M. Z., Murshed, S. M. (2007). Socio-economic determinants of everyday violence in Indonesia: An empirical investigation of Javanese districts. Journal of Peace Research, 44(6), 689-709.

Tauchen, H., Witte, A. D., Griesinger, H. (1994). Criminal deterrence: revisiting the issue with a birth cohort. The Review of Economics and Statistics, 76(3), 399-412.

Teixeira, E. C. (2011). Dois ensaios acerca da relação entre criminalidade e educação. 2011. 102 f. Tese (Doutorado na área de Economia Aplicada) - ESALQ/Universidade de São Paulo, São Paulo.

Waiselfisz, J. J. (coord) (1998). Juventude, Violência e Cidadania: os jovens de Brasília. Brasília: Unesco. 